\title{
CONTINUOUS FAMILY GROUPOIDS
}

\author{
ALAN L. T. PATERSON \\ (communicated by Ronald Brown)
}

\begin{abstract}
In this paper, we define and investigate the properties of continuous family groupoids. This class of groupoids is necessary for investigating the groupoid index theory arising from the equivariant Atiyah-Singer index theorem for families, and is also required in noncommutative geometry. The class includes that of Lie groupoids, and the paper shows that, like Lie groupoids, continuous family groupoids always admit (an essentially unique) continuous left Haar system of smooth measures. We also show that the action of a continuous family groupoid $G$ on a continuous family $G$-space fibered over another continuous family $G$ space $Y$ can always be regarded as an action of the continuous family groupoid $G * Y$ on an ordinary $G * Y$-space.
\end{abstract}

\section{Introduction}

In the context of noncommutative geometry, it is becoming increasingly clear that the classical Atiyah-Singer index theory, in which a compact group acts equivariantly on a compact manifold, requires to be extended to the context of a Lie groupoid acting properly on a manifold. In this connection, Connes ([5, p.151]) says:

One of the interests of the general formulation [of the Baum-Connes conjecture] is to put many particular results in a common framework. Thus, for instance, the following three theorems:

1) The Atiyah-Singer index theorem for covering spaces

2) The index theorem for measured foliations

3) The index theorem for homogeneous spaces

are all special cases of the same index theorem for $G$-invariant elliptic operators $D$ on proper $G$-manifolds, where $G$ is a smooth [i.e. Lie] groupoid with a transverse measure $\Lambda$.

For example, in the foliation case, the smooth (Lie) groupoid $G$ is the holonomy groupoid of a compact foliated manifold. Like all Lie groupoids, it acts properly on itself as a $G$-space, each $g \in G$ acting as a diffeomorphism from $G^{s(g)}$ onto $G^{r(g)}$. (Here, $s, r$ are respectively the source and range maps on the groupoid. See $\S 2$ for more details.) So the "proper $G$-manifold" in that case is $G$ itself. Each $G$-invariant family of elliptic pseudodifferential operators along the leaves defines an element of $K^{0}\left(F^{*}\right)$, where $F$ is the vector bundle of vectors tangential along the $G^{u}$ 's, and has its index in $K_{0}\left(C^{*}(G)\right)$.

We note that smoothness is really only being used along the "leaves" $G^{u}$ (and $G_{u}$ ), and this leads naturally to considering the case where only this kind of smoothness is assumed for the groupoid, and we have only continuity "transversely". (This notion, as well as the $C^{\infty, 0}$ notation of the paper, appears in [4].) In fact, such a class of groupoids is required for the equivariant version of the Atiyah-Singer index theorem for continuous families ([2]) alluded

Received 3 March 2000, revised 17 August 2000; published on 4 September 2000.

2000 Mathematics Subject Classification: Primary: 22A22; 58H05; Secondary 58G10

Key words and phrases: Groupoids, continuous families, continuous left Haar systems, $G$-spaces, index theorems (C) 2000, Alan L. T. Paterson. Permission to copy for private use granted. 
to at the end of that paper $([\mathbf{2}, \mathrm{p} .135])$. (The groupoid interpretation of this theorem is given in $\S 4$ of the present paper.) This equivariant index theorem cannot be formulated in terms of Lie groupoids since the unit space of the groupoid in that context is the base space $Y$ of the continuous family on which a compact Lie group acts, and $Y$ is not assumed to be a manifold but only a compact Hausdorff space. To deal with this, then, we need to consider a class of locally compact groupoids more general than that of Lie groupoids, in which, as above, we only have smoothness along the leaves. The groupoids that we need for this are the continuous family groupoids in the title of the paper.

Continuous families (in the sense of Atiyah and Singer) play an important role in index theory. For example, the Bott periodicity theorem, which asserts (for compact $Y$ ) that $K^{0}(Y)=K^{0}\left(Y \times \mathbb{S}^{2}\right)$, involves the continuous family $Y \times \mathbb{S}^{2}$ over $Y$. So if we restricted only to smooth families, we would be effectively restricting K-theory to smooth manifolds, whereas $\mathrm{K}$-theory is a functor on the topological category.

In the non-equivariant families theorem, a continuous family is defined as follows. We are given a compact Hausdorff space $Y$ and a fiber bundle $X$ over $Y$ with fiber $Z$, where $Z$ is a smooth compact manifold and the structure group of $X$ is $\operatorname{Diff}(Z){ }^{1}(\operatorname{Here} \operatorname{Diff}(Z)$ is a topological group under the topology of uniform convergence for each derivative.) The space $X$ is thus a continuous family of spaces diffeomorphic to $Z$. Atiyah and Singer ([2]) showed that the index of an elliptic family on $X$ lies in $K^{0}(Y)$.

For continuous family groupoids, we need to extend this to the case where the fibers are not diffeomorphic to a fixed space. The general notion of a continuous family of manifolds, required for the paper, is more accurately described as being a locally continuous family $X$ of smooth manifolds $X^{y}$ with $y \in Y$, but for the sake of brevity, we will omit the adjective "locally". (The smooth version of this for almost differentiable groupoids is given in [12].) The role of $\operatorname{Diff}(Z)$ is taken over by a certain pseudogroup of maps. We then (as in [2]) describe what we mean by a vector bundle over $X$ which is smooth along the fibers. (This is not actually used later in the paper but is included since it is required for groupoid index theory.)

The definition of continuous family groupoids is given in §3. Lie groupoids are, of course, continuous family groupoids. But there are many naturally occurring examples of continuous family groupoids that are not Lie groupoids (including, in particular, the groupoid associated with the equivariant index theorem for families referred to earlier). As for Lie groupoids, there is an essentially unique, continuously varying, left Haar system of smooth measures for any continuous family groupoid $G$, so that there is a canonical $C^{*}(G)$. (The $K_{0}$-group of this $C^{*}$-algebra is the recipient for the index of elliptic families on $G$-manifolds.)

The last section $\S 4$ of the paper discusses $G$-spaces. Its main result is that for the category of continuous family groupoids, by changing the groupoid, we need only consider "ordinary" $G$-spaces rather than fibered $G$-spaces. Indeed, suppose that we are given $G$-spaces $X, Y$ with $X$ fibered equivariantly over $Y$. Then we can form the "transformation groupoid" $G * Y$ associated with the action of $G$ on $Y$. This groupoid is shown to be itself a continuous family groupoid, and the action of $G$ on $X$ fibered over $Y$ is equivalent to the canonical action of $G * Y$ on $X$ with $X$ treated as an ordinary $G * Y$-space.

In the case of the equivariant Atiyah-Singer families index theorem, in which a compact Lie group $H$ acts equivariantly on a compact manifold $X$ over $Y$, the preceding shows that this is equivalent to the transformation group groupoid $H * Y$ acting on $X$, and even in that classical context, we leave the group category for the continuous family groupoid category. (The index of a $H$-invariant elliptic family of pseudodifferential operators on $X$ can be shown to lie in $K_{0}\left(C^{*}(H * Y)\right)$.)

\footnotetext{
${ }^{1}$ In the notation of [2], the $X$ and $Z$ are interchanged.
} 


\section{Continuous families of manifolds}

Let $Y$ be a topological space and $k \geqslant 1$. Let $A_{1}, A_{2}$ be open subsets of $Y \times \mathbb{R}^{k}$ such that $q_{1}\left(A_{1}\right) \subset q_{1}\left(A_{2}\right)$ where $q_{1}$ is the canonical projection map from $Y \times \mathbb{R}^{k}$ onto $Y$. For $y \in q_{1}\left(A_{i}\right)$ $(i=1,2)$, let $A_{i}^{y}=\left\{x \in \mathbb{R}^{k}:(y, x) \in A_{i}\right\}$. Let $f: A_{1} \rightarrow A_{2}$ be a continuous map which preserves fibers, i.e. for each $y \in q_{1}\left(A_{1}\right)$, we have $f\left(\{y\} \times A_{1}^{y}\right) \subset\{y\} \times A_{2}^{y}$. For such an $f$, define $f^{y}: A_{1}^{y} \rightarrow A_{2}^{y}$ by: $f(y, x)=\left(y, f^{y}(x)\right)$.

Then (cf. [4, p. 110]) the function $f$ is said to be a $C^{\infty, 0}$-function $\left(f \in C^{\infty, 0}\left(A_{1}, A_{2}\right)\right)$ if, whenever $U_{1}, U_{2}$ are open subsets of $Y$ and $V_{1}, V_{2}$ are open subsets of $\mathbb{R}^{k}$ such that $U_{i} \times V_{i} \subset A_{i}$ for each $i$ and $f\left(U_{1} \times V_{1}\right) \subset U_{2} \times V_{2}$, then the map $y \rightarrow\left(f^{y}\right)_{\mid V_{1}}$ is a continuous map from $U_{1}$ into $C^{\infty}\left(V_{1}, V_{2}\right)$. Here, the topology on $C^{\infty}\left(V_{1}, V_{2}\right)$ is that of uniform convergence on compacta for all derivatives, i.e. $h_{n} \rightarrow h$ in $C^{\infty}\left(V_{1}, V_{2}\right)$ if and only if, for every compact subset $K$ of $V_{1}$ and multi-index $\alpha=\left(\alpha_{1}, \alpha_{2}, \ldots, \alpha_{k}\right)$, we have

$$
\left|\partial^{\alpha} h_{n}(x)-\partial^{\alpha} h(x)\right|_{K} \rightarrow 0
$$

as $n \rightarrow \infty$. Here, the differentiation is with respect to the $x_{i}$ 's where $x=\left(x_{1}, \ldots, x_{k}\right)$, and $|g|_{K}=\sup _{x \in K}|g(x)|$ for any complex-valued function $g$ bounded on $K$.

In the above definition, since $f\left(U_{1} \times V_{1}\right) \subset U_{2} \times V_{2}$ and $f$ is fiber preserving, we have $U_{1} \subset U_{2}$. We can clearly take $U_{1}=U_{2}$ above. In addition, by the continuity of $f$, every element of $A_{1}$ belongs to some open $U_{1} \times V_{1} \subset A_{1}$ for which there exists an open $U_{2} \times V_{2} \subset A_{2}$ with $f\left(U_{1} \times V_{1}\right) \subset U_{2} \times V_{2}$.

The set $\operatorname{Diff}^{0}\left(A_{1}, A_{2}\right)$ is defined to be the set of functions $f \in C^{\infty, 0}\left(A_{1}, A_{2}\right)$ for which $f^{-1}$ exists and belongs to $C^{\infty, 0}\left(A_{2}, A_{1}\right)$. (If $\operatorname{Diff}^{0}\left(A_{1}, A_{2}\right)$ is non-empty, then of course $q_{1}\left(A_{1}\right)=$ $q_{1}\left(A_{2}\right)$.) Every element of $\operatorname{Diff}^{0}\left(A_{1}, A_{2}\right)$ is trivially a homeomorphism.

In practice, an alternative formulation of $C^{\infty, 0}\left(A_{1}, A_{2}\right)$ proves useful. Let us say that a fiber preserving function $f: A_{1} \rightarrow A_{2}$ is $C^{\infty}$-continuous if given $a \in A_{1}$ and an open subset of $A_{2}$ of the form $U_{2} \times V_{2}\left(U_{2} \subset Y, V_{2} \subset \mathbb{R}^{k}\right)$ which contains $f(a)$, then there exists an open subset $U_{1} \times V_{1}$ of $A_{1}$ such that $a \in U_{1} \times V_{1}, f\left(U_{1} \times V_{1}\right) \subset U_{2} \times V_{2}$ and the map $y \rightarrow\left(f^{y}\right)_{\mid V_{1}}$ takes $U_{1}$ into $C^{\infty}\left(V_{1}, V_{2}\right)$.

Proposition 1. A function $f: A_{1} \rightarrow A_{2}$ belongs to $C^{\infty, 0}\left(A_{1}, A_{2}\right)$ if and only if it is $C^{\infty}$ continuous.

Proof. If $f \in C^{\infty, 0}\left(A_{1}, A_{2}\right)$, then trivially $f$ is $C^{\infty}$-continuous. Conversely, suppose that $f$ is $C^{\infty}$-continuous and let $U_{i} \times V_{i}$ be open subsets of $A_{i}$ such that $f\left(U_{1} \times V_{1}\right) \subset U_{2} \times V_{2}$. Let $K$ be a compact subset of $V_{1}$ and $y_{0} \in U_{1}$. Then for each $v \in K$, there exists, by the $C^{\infty}$-continuity of $f$, an open subset $U_{i}^{v} \times V_{i}^{v}$ of $A_{i}$ with $\left(y_{0}, v\right) \in U_{1}^{v} \times V_{1}^{v}$ such that the map $y \rightarrow\left(f^{y}\right)_{\mid V_{1}^{v}}$ is continuous from $U_{1}^{v}$ into $C^{\infty}\left(V_{1}^{v}, V_{2}^{v}\right)$. Cover $K$ by a finite number of sets $V_{1}^{v_{1}}, \ldots, V_{1}^{v_{n}^{1}}$, and let $U^{\prime}=\cap_{j=1}^{n} U_{1}^{v_{j}}$. There exist compact subsets $K^{j}(1 \leqslant j \leqslant n)$ such that $K^{j} \subset V_{1}^{v_{j}}$ and $K=\cup_{j=1}^{n} K^{j}$. Then each of the maps $y \rightarrow\left(f^{y}\right)_{\mid V_{1}^{v_{j}}}$ is continuous, and it follows (using the $K^{j}$ 's) that $\left\|\partial^{\alpha} f^{y}-\partial^{\alpha} f^{y_{0}}\right\|_{K} \rightarrow 0$ if $y \rightarrow y_{0}$ in $U^{\prime}$. So $f \in C^{\infty, 0}\left(A_{1}, A_{2}\right)$.

Corollary 1. Let $A_{1}, A_{2}, A_{3}$ be open subsets of $Y \times \mathbb{R}^{k}$ such that $q_{1}\left(A_{i}\right) \subset q_{1}\left(A_{i+1}\right)(1 \leqslant$ $i \leqslant 2)$. Let $f \in C^{\infty, 0}\left(A_{1}, A_{2}\right)$ and $g \in C^{\infty, 0}\left(A_{2}, A_{3}\right)$. Then $g \circ f \in C^{\infty, 0}\left(A_{1}, A_{3}\right)$. Further, if $f \in \operatorname{Diff}^{0}\left(A_{1}, A_{2}\right)$, then $f^{-1} \in \operatorname{Diff}^{\ominus}\left(A_{1}, A_{2}\right)$.

Proof. For the first assertion of the corollary, one just has to prove that $g \circ f$ is $C^{\infty}$-continuous. To this end, note that $(g \circ f)^{y}=g^{y} \circ f^{y}$. One then follows the elementary proof that the composition of two continuous functions is continuous, and uses induction and the chain rule to deal with the partial derivatives in (2.1). The second assertion is obvious.

We now recall the definition of a pseudogroup. Various definitions have been given of this in the literature: the version that we will use is that given in [6, p.1]. A pseudogroup $S$ on a topological space $X$ is an inverse semigroup of homeomorphisms $f: A \rightarrow B$, where $A, B$ are open subsets of $X$ (depending on $f$ ) such that: 
(i) if $f: A \rightarrow B$ is a homeomorphism, where $A=\cup_{i \in I} A_{i}$ and $f_{\mid A_{i}} \in S$, then $f \in S$;

(ii) if $f: A \rightarrow B$ belongs to $S$ and $A^{\prime}$ is an open subset of $A$, then $f_{\mid A^{\prime}} \in S$.

(iii) If $A$ is open in $X$, then the identity map id : $A \rightarrow A$ belongs to $S$.

Let $\Gamma\left(Y \times \mathbb{R}^{k}\right)$ be the union of all of the sets $\operatorname{Diff}^{0}\left(A_{1}, A_{2}\right)$ (with $A_{1}, A_{2}$ ranging over the open subsets of $\left.Y \times \mathbb{R}^{k}\right)$.

Proposition 2. The set $\Gamma\left(Y \times \mathbb{R}^{k}\right)$ is a pseudogroup on $Y \times \mathbb{R}^{k}$.

Proof. Let $S=\Gamma\left(Y \times \mathbb{R}^{k}\right)$. By Corollary 1, if $f \in \operatorname{Diff}^{0}\left(A_{1}, A_{2}\right)$ and $g \in \operatorname{Diff}^{0}\left(A_{3}, A_{4}\right)$, then $f^{-1} \in S$ and both $f \circ g,(f \circ g)^{-1}=g^{-1} \circ f^{-1}$ are Diff ${ }^{0}$ maps. From Corollary 1, if $f \in \operatorname{Diff}^{0}\left(A_{1}, A_{2}\right)$ and $g \in \operatorname{Diff}^{0}\left(A_{3}, A_{4}\right)$ then $g \circ f \in \operatorname{Diff}^{0}\left(A_{5}, A_{6}\right)$, where $A_{5}=f^{-1}\left(A_{2} \cap A_{3}\right)$ and $A_{6}=g\left(A_{2} \cap A_{3}\right)$. So $f^{-1}, f \circ g \in S$, and $S$ is an inverse semigroup. Conditions (i) and (ii) above follow by $C^{\infty}$-continuity (cf. the proof of Proposition 1), while (iii) is trivial.

Let $X, Y$ be locally compact Hausdorff spaces and $p: X \rightarrow Y$ be a continuous open surjection. We say that $(X, p)$ is fibered over $Y$ with fibers $X^{y}=p^{-1}(\{y\})(y \in Y)$, and call $(X, p)$ a fiber space (over $Y)$. We now define what is meant by a continuous family of manifolds over $Y$.

Definition 1. Let $(X, p)$ be fibered over $Y$. Then the pair $(X, p)$ is defined to be a continuous family of manifolds $X^{y}$ over $Y$ or simply a continuous family over $Y$ if there exists a set of pairs $\left\{\left(U_{\alpha}, \phi_{\alpha}\right): \alpha \in A\right\}$, where each $U_{\alpha}$ is an open subset of $X$ and $\cup_{\alpha \in A} U_{\alpha}=X$, compatible with the pseudogroup $\Gamma\left(Y \times \mathbb{R}^{k}\right)$ in the following sense:

(i) for each $\alpha$, the map $\phi_{\alpha}$ is a homeomorphism from $U_{\alpha}$ onto an open subset of $Y \times \mathbb{R}^{k}$ for which $q_{1} \circ \phi_{\alpha}=p_{\mid U_{\alpha}}$;

(ii) for each $\alpha, \beta$, the mapping $\phi_{\beta} \circ \phi_{\alpha}^{-1} \in \operatorname{Diff}^{\rho}\left(\phi_{\alpha}\left(U_{\alpha} \cap U_{\beta}\right), \phi_{\beta}\left(U_{\alpha} \cap U_{\beta}\right)\right)$.

The family $\mathfrak{A}=\left\{U_{\alpha}: \alpha \in A\right\}$ will be called an atlas for the continuous family $(X, p)$, and the $U_{\alpha}$ 's, or more precisely, the pairs $\left(U_{\alpha}, \phi_{\alpha}\right)$, will be called charts. Of course, in the above definition, we can and will take the atlas $\mathfrak{A}$ to be maximal. Then $\mathfrak{A}$ is a basis for the topology of $X$.

If $(U, \phi) \in \mathfrak{A}$ and $x \in U$, then there exists a $V \subset U$ with $x \in V$ such that $\left(V,\left.\phi\right|_{V}\right) \in \mathfrak{A}$ and $\phi(V)=p(V) \times W$ for some open subset $W$ of $\mathbb{R}^{k}$.805We shall write $V \sim p(V) \times W$. For many purposes, we need only consider charts of this special form $V$.

The simplest example of a continuous family over $Y$ is one of the form $X=Y \times M$ where $M$ is a manifold. Such a family is called trivial. From the preceding paragraph, every continuous family is locally trivial.

A continuous family in the sense of Atiyah and Singer is a continuous family in our sense. To see this, recall $(\S 1)$ that in that case, $(X, p)$ is a fiber bundle over $Y$ with a manifold $Z$ as fiber and with structure group $\operatorname{Diff}(Z)$. Then there is a basis $\left\{J_{\delta}\right\}$ for the topology of $Y$ and fiber preserving homeomorphisms $c_{\delta}: p^{-1}\left(J_{\delta}\right) \rightarrow J_{\delta} \times Z$ such that the resultant cocycles are continuous maps into $\operatorname{Diff}(Z)$. Let $\left(L_{\gamma}, \chi_{\gamma}\right)$ be a chart for $Z$. We obtain charts for the continuous family in the sense of Definition 1 by taking sets of the form $\left(\left(1 \otimes \chi_{\gamma}\right) \circ c_{\delta}\right)^{-1}(A)$ where $A$ is an open subset of $J_{\delta} \times \mathbb{R}^{k}(k=\operatorname{dim} Z)$..

Smooth families are, of course, continuous families. These arise in the theory of Lie groupoids $(\S 3)([\mathbf{8}, \mathbf{1 2}, \mathbf{1 3}, \mathbf{1 5}, \mathbf{1 6}])$. (In particular, for any Lie groupoid $G$ with range and source maps $r, s$ and unit space $G^{0}$, both $(G, r),(G, s)$ are smooth families over $G^{0}$.) For a smooth family, we require that both $X, Y$ be manifolds and that $p: X \rightarrow Y$ be a (surjective) submersion. Then locally, $p$ can be taken to be a smooth projection map $(x, y) \rightarrow x$, and thus defines a foliated manifold structure on $X$ whose leafs are the $X^{y}$ 's ([3, p.23-24]). Condition (ii) of Definition 1 is satisfied since the maps $\phi_{\beta} \circ \phi_{\alpha}^{-1}$ are diffeomorphisms. 
Let $(X, p)$ be a continuous family of manifolds with atlas $\mathfrak{A}=\left\{U_{\alpha}: \alpha \in A\right\}$. Then (as is to be expected) every $X^{y}$ is a (smooth) manifold. Indeed, for fixed $y$, let

$$
\mathfrak{A}^{y}=\left\{U_{\alpha} \cap X^{y}: \alpha \in A\right\} .
$$

Then $\mathfrak{A}^{y}$ gives the relative topology on $X^{y}$ (as a closed subset of $X$ ). Since the restriction of $\phi_{\beta} \circ \phi_{\alpha}^{-1}$ to $\phi_{\alpha}\left(U_{\alpha} \cap U_{\beta} \cap X^{y}\right)$ is a diffeomeomorphism onto $\phi_{\beta}\left(U_{\alpha} \cap U_{\beta} \cap X^{y}\right)$, we obtain that $X^{y}$ is a manifold.

We now describe some operations that produce new continuous families from given ones. We note without giving details that similar (easier) constructions can be given for fiber spaces.

A pull-back of a continuous family is also a continuous family. Specifically, let $(X, p)$ be a continuous family over $Y, Z$ be a locally compact Hausdorff space and $t: Z \rightarrow Y$ be a continuous map. The pull-back continuous family $\left(t^{-1} X, p^{\prime}\right)$ over $Z$ is given by the subset

$$
t^{-1} X=\{(z, x) \in A \times X: t(z)=p(x)\}
$$

of $Z \times X$ and the map $p^{\prime}$ where $p^{\prime}((z, x))=z$. We have the commuting diagram:

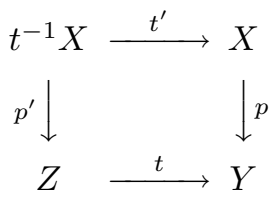

where $t^{\prime}((z, x))=x$. We obtain charts for $\left(t^{-1} X, Z\right)$ as follows. If $(U, \phi)$ is a chart for $X$ and $\phi(x)=(p(x), h(x))$, then $\left(t^{-1} U, \phi^{\prime}\right)$ is a chart for $t^{-1} X$, where

$$
\phi^{\prime}((z, x))=(z, h(x)) .
$$

Let $\left(X_{1}, p_{1}\right),\left(X_{2}, p_{2}\right)$ be continuous families over the same space $Y$ and $f: X_{1} \rightarrow X_{2}$ be a continuous fiber preserving map, i.e. $p_{2} \circ f=p_{1}$. We say that $f \in C^{\infty, 0}\left(X_{1}, X_{2}\right)$ if $f$ is locally $C^{\infty, 0}$ in the earlier sense. That is, whenever $(U, \phi),(V, \psi)$ are charts for $X_{1}$ and $X_{2}$ respectively such that $p_{1}(U)=p_{2}(V)$ and $f(U) \subset V$, then $\psi \circ f \circ \phi^{-1} \in C^{\infty, 0}(\phi(U), \psi(V))$.

We now discuss what is meant by a morphism of continuous families. We deal first with a special case (to which, as we shall see, the general case can be reduced). In the special case, a morphism from $\left(X_{1}, p_{1}\right)$ into $\left(X_{2}, p_{2}\right)$ over $Y$ as in the preceding paragraph is just a function $f \in C^{\infty, 0}\left(X_{1}, X_{2}\right)$. We represent such a morphism by the commutative diagram:

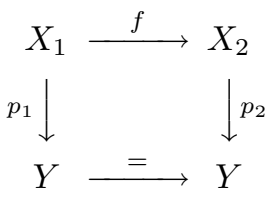

We now define what is meant by a morphism of continuous families in the general case. Let $\left(X_{1}, p_{1}\right),\left(X_{2}, p_{2}\right)$ be continuous families over locally compact Hausdorff spaces $Y_{1}, Y_{2}$. Let $q: Y_{1} \rightarrow Y_{2}$ be a continuous map and $f: X_{1} \rightarrow X_{2}$ be a continuous fiber preserving map with respect to $q$ in the sense that $p_{2} \circ f=q \circ p_{1}$. (In the special case of a morphism above, $Y_{1}=Y_{2}=Y$ and $q$ is the identity map.) For $y \in Y_{1}$, let $f^{y}: X_{1}^{y} \rightarrow X_{2}^{q(y)}$ be the restriction of $f$ to $X_{1}^{y}$. Then $f$ is called a morphism (with respect to $q$ ) if for each $y \in Y_{1}$, the function $f^{y} \in C^{\infty}\left(X_{1}^{y}, X_{2}^{q(y)}\right)$ and the map $y \rightarrow f^{y}$ is locally a $C^{\infty, 0}$-function. More precisely, given $x \in X_{1}$ and a chart $U_{2} \sim p_{2}\left(U_{2}\right) \times W_{2}$ in $X_{2}$ containing $f(x)$, then there exists a chart $U_{1} \sim p_{1}\left(U_{1}\right) \times W_{1}$ in $X_{1}$, and such that $x \in U_{1}, f\left(U_{1}\right) \subset U_{2}$ and (in local coordinates) the map $y \rightarrow f^{y}$ is continuous from $p_{1}\left(U_{1}\right)$ into $C^{\infty}\left(W_{1}, W_{2}\right)$ where

$$
f\left(\left(y, w_{1}\right)\right)=\left(q(y), f^{y}\left(w_{1}\right)\right) .
$$

We think of the continuous family $X_{1}$ over $Y_{1}$ as being taken over into the continuous family $X_{2}$ over $Y_{2}$ by the maps $f, q$. 
We represent a morphism $f$ by the following commutative diagram:

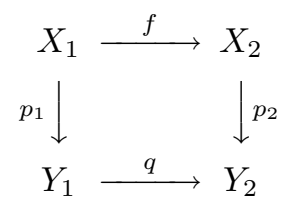

The set of morphisms $f$ from $X_{1}$ into $X_{2}$ is denoted by $C^{\infty, 0}\left(X_{1}, X_{2}\right)$.

It is easy to prove that if $\left(X_{3}, p_{3}\right)$ is a continuous family over $Y_{3}, q^{\prime}: Y_{2} \rightarrow Y_{3}$ is continuous and $g: X_{2} \rightarrow X_{3}$ is a morphism with respect to $q^{\prime}$, then $g \circ f: X_{1} \rightarrow X_{3}$ is a morphism with respect to $q^{\prime} \circ q$. The commuting diagram for the morphism $g \circ f$ can be represented as the "product" of the following commuting diagram:

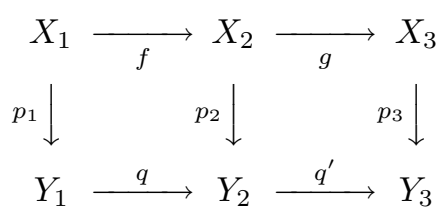

It is easy to show that the class of continuous families is a category with morphisms $f$ as above.

A morphism $f: X_{1} \rightarrow X_{2}$ in the general case can be reduced simply to the special case by using a pull-back continuous family. More precisely, the pull-back continuous family $q^{-1} X_{2}$ is a continuous family over $Y_{1}$, and we have a map $f^{\prime}: X_{1} \rightarrow q^{-1} X_{2}$ given by: $f^{\prime}(x)=\left(p_{1}(x), f(x)\right)$. It is left to the reader to check that a continuous, fiber-preserving map $f$ is a morphism if and only if $f^{\prime}$ is a (special case) morphism.

In the case where $X=X_{1}, Y=Y_{1}, X_{2}=\mathbb{C}$ and $Y_{2}$ is a singleton, then we write $C^{\infty, 0}\left(X_{1}, \mathbb{C}\right)=C^{\infty, 0}\left(X_{1}\right)$. A simple argument (using the charts for $X$ ) shows that $C^{\infty, 0}(X)$ is a ${ }^{*}$-subalgebra of $C(X)$, the algebra of continuous complex-valued functions on $X$. It is left to the reader to show that $X$ admits $C^{\infty, 0}$ - partitions of unity.

Let $C_{c}^{0, \infty}(X)$ be the subalgebra of functions with compact support in $C^{\infty, 0}(X)$. If $X$ is a product $Y \times V$ where $V$ is an open subset of $\mathbb{R}^{k}$ and $\left(y_{0}, k_{0}\right) \in X$, then there is a function $f \in C_{c}^{0, \infty}(X)$ with $f\left(\left(y_{0}, k_{0}\right)\right)=1$. Indeed, we can take $f=g \otimes h$ where $g \in C_{c}(Y), h \in C_{c}^{\infty}(V)$ and $g\left(y_{0}\right)=1=h\left(k_{0}\right)$. By considering charts, it follows that for a general continuous family $X$, the space $C_{c}^{0, \infty}(X)$ separates the points of $X$.

Now let $\left(X_{1}, p_{1}\right),\left(X_{2}, p_{2}\right)$ be continuous families over $Y$. Let $\left(X_{1} * X_{2}, p\right)$ be the fibered product of $\left(X_{1}, p_{1}\right)$ and $\left(X_{2}, p_{2}\right)$ : so

$$
X_{1} * X_{2}=\left\{\left(x_{1}, x_{2}\right) \in X_{1} \times X_{2}: p_{1}\left(x_{1}\right)=p_{2}\left(x_{2}\right)\right\}
$$

and $p\left(x_{1}, x_{2}\right)=p_{1}\left(x_{1}\right)=p_{2}\left(x_{2}\right)$. We sometimes write $p=p_{1} * p_{2}$. Then with the relative topology, $\left(X_{1} * X_{2}, p\right)$ is a continuous family of manifolds over $Y$, with each fiber $X^{y}=$ $X_{1}{ }^{y} \times X_{2}{ }^{y}$ having the product manifold structure. Indeed, it is left to the reader to check that if $\left(x_{1}, x_{2}\right) \in X_{1} * X_{2}$ and $\left(U_{i}, \phi_{i}\right)$ are charts for $X_{i}(i=1,2), x_{i} \in U_{i}, p_{1}\left(U_{1}\right)=p_{2}\left(U_{2}\right)$, then in an obvious notation, $\left(U_{1} * U_{2}, \phi_{1} * \phi_{2}\right)$ is a chart for $X$ and these charts determine an atlas for $X_{1} * X_{2}$. Further, $p$ is a continuous surjection which is open since $p\left(U_{1} * U_{2}\right)=p_{1}\left(U_{1}\right)$ is open in $Y$.

For clarity, we will sometimes write $\left(X_{1}, p_{1}\right) *\left(X_{2}, p_{2}\right)$ in place of $X_{1} * X_{2}$. Note that $Y$ is trivially a continuous family over itself and that $Y * X_{1} \cong X_{1}$. Note also that if $X_{1}^{\prime}, X_{2}^{\prime}$ are continuous families over $Y$ and if $a_{i}: X_{i}^{\prime} \rightarrow X_{i}$ are morphisms, then the natural map $a_{1} * a_{2}: X_{1}^{\prime} * X_{2}^{\prime} \rightarrow X_{1} * X_{2}$ is a morphism of continuous families. This map is an isomorphism if both $a_{1}, a_{2}$ are. These are proved by reducing to the case of charts.

We will need a slight generalization of $X_{1} * X_{2}$ above later. In this situation, we have, for $i=1$, 2 , fiber spaces $\left(Y_{i}, v_{i}\right)$ over some $Z$, and $\left(X_{i}, p_{i}\right)$ continuous families over $Y_{i}$. So $Y_{1} * Y_{2}$ is a fiber space over $Z$. We can then form the continuous family $\left(X_{1} * X_{2}, p_{1} * p_{2}\right)$ over $Y_{1} * Y_{2}$, 
where

$$
X_{1} * X_{2}=\left\{\left(x_{1}, x_{2}\right): x_{i} \in X_{i}, p_{1} * p_{2}\left(x_{1}, x_{2}\right) \in Y_{1} * Y_{2}\right\}
$$

and $p_{1} * p_{2}\left(x_{1}, x_{2}\right)=\left(p_{1}\left(x_{1}\right), p_{2}\left(x_{2}\right)\right.$. Note that when $Y_{1}=Y_{2}=Y=Z$ with each $v_{i}$ the identity map, then the two definitions of $\left(X_{1} * X_{2}, p_{1} * p_{2}\right)$ coincide. It is left to the reader to show that $\left(X_{1} * X_{2}, p_{1} * p_{2}\right)$ is a continuous family over $Y_{1} * Y_{2}$.

Now let $\left(X_{1}, p_{1}\right)$ and $\left(X_{2}, p_{2}\right)$ be continuous families over $Y$. There are two other ways in which $X_{1} * X_{2}$ can be naturally regarded as a continuous family. These give pull-back families $\left(X_{1} * X_{2}, t_{1}\right)$ over $X_{1}$ and $\left(X_{1} * X_{2}, t_{2}\right)$ over $X_{2}$. Here the $t_{i}$ 's are the natural projection maps:

$$
t_{1}\left(x_{1}, x_{2}\right)=x_{1}, t_{2}\left(x_{1}, x_{2}\right)=x_{2} .
$$

Firstly, the pull-back of the continuous family $\left(X_{2}, p_{2}\right)$ by $p_{1}: X_{1} \rightarrow Y$ gives the continuous family $\left(p_{1}^{-1}\left(X_{2}\right), t_{1}\right)$, where

$$
p_{1}^{-1}\left(X_{2}\right)=\left\{\left(x_{1}, x_{2}\right): p_{1}\left(x_{1}\right)=p_{2}\left(x_{2}\right)\right\}=X_{1} * X_{2} .
$$

In the same way, $\left(p_{2}^{-1}\left(X_{1}\right), t_{2}\right)=\left(X_{2} * X_{1}, t_{1}\right)$ over $X_{2}$. Interchanging first and second components gives the continuous family $\left(X_{1} * X_{2}, t_{2}\right)$.

Note that if $\left(X_{2}, p_{2}\right)$ is assumed to be only a fiber space over $Y$ (with $\left(X_{1}, p_{1}\right)$ still being assumed to be a continuous family), then $\left(X_{1} * X_{2}, t_{2}\right)$ is still a continuous family, the $X_{2}$ just playing the continuous role of a parameter space. The following proposition will be used in $\S 4$.

Proposition 3. Let $\left(X_{1}, q_{1}\right)$ and $\left(X_{2}, q_{2}\right)$ be continuous families over $Z$ and $f: X_{1} \rightarrow X_{2}$ be a morphism. Let $(Y, q)$ be a continuous family over $Z$. Then there is a canonical morphism $f * 1$ from $\left(X_{1} * Y, t_{2}\right)$ into $\left(X_{2} * Y, t_{2}\right)$ :

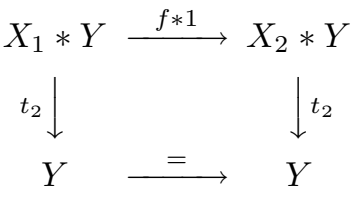

Proof. To obtain (2.5), we "*" the morphism diagram for $f$ by $Y$ to get:

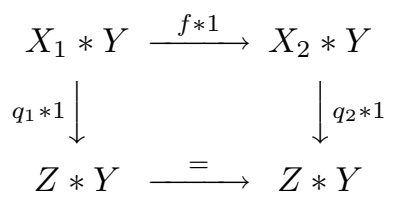

It is easy to check that (2.6) is commutative, and it is left to the reader to check, using charts, that $f * 1$ is a morphism. (2.5) now follows by identifying $Z * Y$ with $Y$ and $q_{i} * 1$ with $t_{2}$.

Now let $(X, p)$ be a continuous family of manifolds over $Y$. We will define what is meant by a smooth vector bundle over $X$ of dimension $m$. This generalizes the corresponding notion in $[\mathbf{2}]$.

We first define the pseudogroup in this situation corresponding to $\Gamma\left(Y \times \mathbb{R}^{k}\right)$ earlier. Let $A_{1}, A_{2}$ be open subsets of $Y \times \mathbb{R}^{k}$ as earlier. Let $m \geqslant 0$ and $B_{i}=A_{i} \times \mathbb{R}^{m}$. Note that each $B_{i} \subset Y \times \mathbb{R}^{k+m}$. Let $f: A_{1} \rightarrow A_{2}$ and $g: B_{1} \rightarrow \mathbb{R}^{m}$, and define $F: B_{1} \rightarrow B_{2}$ by:

$$
F(y, w, \xi)=(y, f(y, w), g(y, w, \xi))
$$

where $(y, w) \in A_{1}, \xi \in \mathbb{R}^{m}$. We say that $F \in \operatorname{Diff}_{\ell}^{0}\left(B_{1}, B_{2}\right)$ if $F \in \operatorname{Diff}^{0}\left(B_{1}, B_{2}\right)\left(B_{1}, B_{2}\right.$ regarded as subsets of $\left.Y \times \mathbb{R}^{m+k}\right)$ and for fixed $(y, w)$, the map $\xi \rightarrow g(y, w, \xi)$ is a vector space isomorphism of $\mathbb{R}^{m}$. Let $\Gamma\left(Y \times \mathbb{R}^{k} ; \mathbb{R}^{m}\right)$ be the union of all of the sets $\operatorname{Diff}_{\ell}^{0}\left(B_{1}, B_{2}\right)$. It is easy to check that $\operatorname{Diff}_{\ell}^{0}\left(B_{1}, B_{2}\right)$ is a subpseudogroup of $\Gamma\left(Y \times \mathbb{R}^{k+m}\right)$.

Definition 2. An m-dimensional vector bundle $(E, \pi)$ over $X$ is said to be a smooth vector bundle if there exists an atlas of charts $\left\{\left(U_{\alpha}, \phi_{\alpha}\right)\right\}$ for $(X, p)$ as in Definition 1 such that: 
(i) for each $\alpha$, there is given a trivialization $\left(\pi^{-1}\left(U_{\alpha}\right), \Psi_{\alpha}\right)$ of $E \mid U_{\alpha}$;

(ii) with $\Psi_{\alpha}$ as in (i) and with $\kappa_{\alpha}=\left(\phi_{\alpha} \otimes 1\right) \Psi_{\alpha}: \pi^{-1}\left(U_{\alpha}\right) \rightarrow Y \times \mathbb{R}^{k+m}$, each of the maps $\kappa_{\beta} \kappa_{\alpha}^{-1}$ belongs to Diff ${ }_{\ell}\left(\kappa_{\alpha}\left(\pi^{-1}\left(U_{\alpha}\right)\right), \kappa_{\beta}\left(\pi^{-1}\left(U_{\beta}\right)\right)\right)$.

It is left to the reader to check that the standard operations on vector bundles (such as those of forming alternating and tensor products of bundles) preserve the smoothness property.

An important smooth vector bundle over $X$ is the tangent bundle $(T X, \pi)$, where

$$
T X=\cup_{y \in Y} T X^{y}
$$

and $\pi$ is the canonical projection map. Let us show that $T X$ is indeed a smooth $k$-dimensional vector bundle over $X$. Let $\left\{\left(U_{\alpha}, \phi_{\alpha}\right): \alpha \in A\right\}$ be an atlas for $(X, p)$ and let $Z_{\alpha}=\pi^{-1}\left(U_{\alpha}\right)$ for each $\alpha$. For each $y \in p\left(U_{\alpha}\right)$, the restriction map $\phi_{\alpha}^{y}$ of $\phi_{\alpha}$ to $X_{\alpha}^{y}=X^{y} \cap U_{\alpha}$ is a diffeomorphism onto an open subset of $\mathbb{R}^{k}$. Define $\Psi_{\alpha}: Z_{\alpha} \rightarrow U_{\alpha} \times \mathbb{R}^{k}$ by:

$$
\Psi_{\alpha}(\gamma)=\left(\phi_{\alpha}(\pi(\gamma)), D_{\pi(\gamma)} \phi_{\alpha}^{p \circ \pi(\gamma)}(\gamma)\right)
$$

It is easily checked that each $\Psi_{\alpha}$ is a bijection onto a set of the form $A \times \mathbb{R}^{k}$ where $A$ is an open subset of $Y \times \mathbb{R}^{k}$, and that every $\Psi_{\alpha}\left(Z_{\alpha} \cap Z_{\beta}\right)$ is also an open subset of this form. The transition function $\Psi_{\beta} \circ \Psi_{\alpha}^{-1}: \Psi_{\alpha}\left(Z_{\alpha} \cap Z_{\beta}\right) \rightarrow \Psi_{\beta}\left(Z_{\alpha} \cap Z_{\beta}\right)$ is given by:

$$
(y, w, \xi) \rightarrow\left(\left(\phi_{\beta}^{y} \circ\left(\phi_{\alpha}^{y}\right)^{-1}\right)(y, w), D_{w}\left(\phi_{\beta}^{y} \circ\left(\phi_{\alpha}^{y}\right)^{-1}\right)(\xi)\right) .
$$

It is routine to check that $\Psi_{\beta} \circ \Psi_{\alpha}^{-1} \in \Gamma\left(Y \times \mathbb{R}^{k} ; \mathbb{R}^{k}\right)$. So $T X$ is a smooth vector bundle over $X$.

Let $E$ be a smooth vector bundle over $X$. A Riemannian metric for $E$ is a family of Riemannian metrics on the smooth vector bundles $E \mid X^{y}(y \in Y)$ which, in terms of local coordinates, vary continuously. Using a $C^{\infty, 0}$ - partition of unity, a standard argument shows that $E$ admits a Riemannian metric.

\section{Continuous family groupoids}

We now discuss the class of locally compact groupoids with which this paper is primarily concerned and which generalize Lie groupoids. We first recall some facts about locally compact groupoids.

A groupoid is most simply defined as a small category with inverses. Spelled out axiomatically, a groupoid is a set $G$ together with a subset $G^{2} \subset G \times G$, a product map $m: G^{2} \rightarrow G$, where we write $m(a, b)=a b$, and an inverse map $i: G \rightarrow G$, where we write $i(a)=a^{-1}$ and where $\left(a^{-1}\right)^{-1}=a$, such that:

(i) if $(a, b),(b, c) \in G^{2}$, then $(a b, c),(a, b c) \in G^{2}$ and

$$
(a b) c=a(b c)
$$

(ii) $\left(b, b^{-1}\right) \in G^{2}$ for all $b \in G$, and if $(a, b)$ belongs to $G^{2}$, then

$$
a^{-1}(a b)=b \quad(a b) b^{-1}=a .
$$

We define the range and source maps $r: G \rightarrow G^{0}, s: G \rightarrow G^{0}$ by:

$$
r(x)=x x^{-1} \quad s(x)=x^{-1} x .
$$

The unit space $G^{0}$ is defined to be $r(G)=s(G)$, or equivalently, the set of idempotents $u$ in $G$. The maps $r, s$ fiber the groupoid $G$ over with fibers $\left\{G^{u}\right\},\left\{G_{u}\right\}$, where $G^{u}=r^{-1}(\{u\})$ and $G_{u}=s^{-1}(\{u\})$. Note that $(x, y) \in G^{2}$ if and only if $s(x)=r(y)$. 
For detailed discussions of groupoids (including locally compact and Lie groupoids below), the reader is referred to the books $[\mathbf{8}, \mathbf{1 3}, \mathbf{1 8}]$. Important examples of groupoids are given by transformation group groupoids and equivalence relations.

A locally compact groupoid is a groupoid $G$ which is also a second countable locally compact Hausdorff space for which multiplication and inversion are continuous. (A detailed discussion of non-Hausdorff locally compact groupoids is given in [13].) Note that $G^{2}, G^{0}$ are closed subsets of $G \times G, G$ respectively. Further, since $r, s$ are continuous, every $G^{u}, G_{u}$ is a closed subset of $G$.

A locally compact groupoid $G$ is called a Lie groupoid if $G$ is a manifold such that:

(i) $G^{0}$ is a submanifold of $G$;

(ii) the maps $r, s: G \rightarrow G^{0}$ are submersions;

(iii) the product and inversion maps for $G$ are smooth.

Note that $G^{2}$ is naturally a submanifold of $G \times G$ and every $G^{u}, G_{u}$ is a submanifold of $G$. (See $[\mathbf{1 3}$, pp.55-56].)

For analysis on a locally compact groupoid $G$, it is essential to have available a left Haar system. This is the groupoid version of a left Haar measure, though unlike left Haar measure on a locally compact group, such a system may not exist and if it does, it will not usually be unique. However, in many case, there is a natural choice of left Haar system. For Lie groupoids, such a system exists and is essentially unique. As we will see later, this result extends to continuous family groupoids defined below.

A left Haar system on a locally compact groupoid $G$ is a family of measures $\left\{\lambda^{u}\right\}\left(u \in G^{0}\right)$, where each $\lambda^{u}$ is a positive regular Borel measure on the locally compact Hausdorff space $G^{u}$, such that the following three axioms are satisfied:

(i) the support of each $\lambda^{u}$ is the whole of $G^{u}$;

(ii) for any $g \in C_{c}(G)$, the function $g^{0}$, where

$$
g^{0}(u)=\int_{G^{u}} g d \lambda^{u}
$$

belongs to $C_{c}\left(G^{0}\right)$;

(iii) for any $x \in G$ and $f \in C_{c}(G)$,

$$
\int_{G^{d(x)}} f(x z) d \lambda^{d(x)}(z)=\int_{G^{r(x)}} f(y) d \lambda^{r(x)}(y) .
$$

The existence of a left Haar system on $G$ has topological consequences for $G$ - it entails that both $r, s: G \rightarrow G^{0}$ are open maps ([13, p.36]).

Definition 3. A locally compact groupoid $G$ is called a continuous family groupoid if:

(i) both $(G, s),(G, r)$ are continuous families of manifolds over $G^{0}$;

(ii) the inversion map $i:(G, s) \rightarrow(G, r)$, where $i(x)=x^{-1}$, is an isomorphism of continuous families of manifolds:

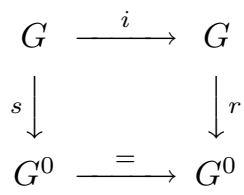

(iii) the multiplication map $m:\left(G * G, t_{1}\right) \rightarrow(G, r)$ is a morphism of continuous families with respect to $r$ :

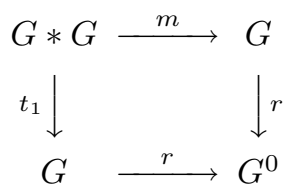


In (iii) above, $G * G$ is defined to be $(G, s) *(G, r)$, so that

$$
G * G=\{(x, y) \in G \times G: s(x)=r(y)\}=G^{2} .
$$

As in $\S 2, G * G$ is a continuous family over $G$ with $t_{1}((x, y))=x$. The map $m$ is a fiber preserving map from $\left(G * G, t_{1}\right)$ into $(G, r)$ (with respect to $\left.r: G \rightarrow G^{0}\right)$ since $r(x y)=r(x)$.

Condition (iii) as stated is one-sided in that the morphism property of $m$ is formulated in terms of $t_{1}, r$ rather than $t_{2}, s$. Indeed, there is a commuting diagram:

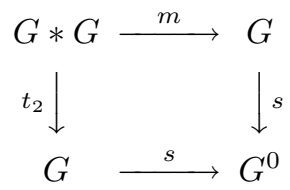

and it is as natural to formulate the morphism property for $m$ in terms of (3.3) as in terms of (3.2). We now show that these two formulations are actually equivalent given (i) and (ii) of Definition 3.

Proposition 4. Let $G$ be a locally compact groupoid satisfying (i) and (ii) of Definition 3. Then the multiplication map $m:\left(G * G, t_{1}\right) \rightarrow(G, r)$ is a morphism with respect to $r$ if and only if $m:\left(G * G, t_{2}\right) \rightarrow(G, s)$ is a morphism with respect to $s$.

Proof. We show first that $(i * i)^{\tilde{y}}$, where $(i * i)^{\tilde{(}}((x, y))=\left(y^{-1}, x^{-1}\right)$, is a morphism from $\left(G * G, t_{1}\right)$ to $\left(G * G, t_{2}\right)$ with respect to $i$ :

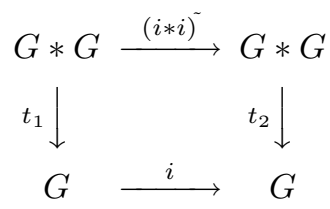

Clearly, $(i * i)^{\tilde{2}}$ is fiber preserving with respect to $i$. Let $(x, y) \in G * G$. Let $U^{\prime}$ be a chart for $\left(G * G, t_{2}\right)$ containing $\left(y^{-1}, x^{-1}\right)$. We can suppose that $U^{\prime}=U_{1} * U_{2}$ where $U_{1}$ is a chart for $(G, s), U_{2}$ is a chart for $(G, r)$ and $s\left(U_{1}\right)=r\left(U_{2}\right)$. We can suppose further that $U_{1} \sim$ $s\left(U_{1}\right) \times W_{1}, U_{2} \sim r\left(U_{2}\right) \times W_{2}$. Then $U_{1} * U_{2} \sim U_{2} \times W_{1}$ under the map $\left(u_{1}, u_{2}\right) \rightarrow\left(u_{2}, w_{1}\right)$ where $u_{1} \sim\left(s\left(u_{1}\right), w_{1}\right)$. Note that $((i * i))^{-1}\left(U_{1} * U_{2}\right)=U_{2}^{-1} * U_{1}^{-1}$.

By (ii) of Definition 3, there exists a chart $U_{1}^{\prime}$ for $(G, s)$ with $x \in U_{1}^{\prime}$ and $U_{1}^{\prime} \sim s\left(U_{1}^{\prime}\right) \times$ $W_{1}^{\prime}, i\left(U_{1}^{\prime}\right) \subset U_{2}$. Similarly, there exists a chart $U_{2}^{\prime}$ for $(G, r)$ with $y \in U_{2}^{\prime}$ and $U_{2}^{\prime} \sim r\left(U_{2}^{\prime}\right) \times$ $W_{2}^{\prime}, i\left(U_{2}^{\prime}\right) \subset U_{1}$. Since $s(x)=r(y)$, we can, by contracting $U_{1}^{\prime}, U_{2}^{\prime}$, suppose that $s\left(U_{1}^{\prime}\right)=r\left(U_{2}^{\prime}\right)$. Then $(x, y) \in U_{1}^{\prime} * U_{2}^{\prime} \sim U_{1}^{\prime} \times W_{2}^{\prime}$ is a chart for $\left(G * G, t_{1}\right)$. Now in local terms, for $\left(x_{1}, w_{2}^{\prime}\right) \in$ $U_{1}^{\prime} \times W_{2}^{\prime}$,

$$
(i * i)^{\tilde{(}}\left(x_{1}, w_{2}^{\prime}\right)=\left(i^{-1}\left(s\left(x_{1}\right), w_{2}^{\prime}\right), x_{1}^{-1}\right)
$$

so that $(i * i)^{\tilde{}}$ is a morphism, again using (ii) of Definition 3.

Suppose that $m:\left(G * G, t_{2}\right) \rightarrow(G, s)$ is a morphism with respect to $s$. Then $m:\left(G * G, t_{1}\right) \rightarrow$ $(G, r)$ is a morphism since it is the morphism product $i \circ m \circ(i * i)^{\tilde{2}}$ :

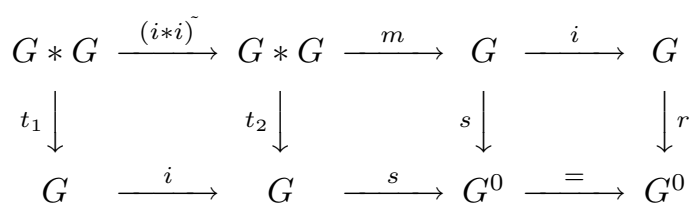

The converse is true in a similar way.

Let $G$ be a continuous family groupoid. Note that $(G * G)^{x}=\{x\} \times G^{s(x)}$ in $\left(G * G, t_{1}\right)$, so that what (iii) of Definition 3 above is saying is that for fixed $x \in G$, the map $L_{x}: y \rightarrow x y$ is a diffeomorphism from $G^{s(x)}$ onto $G^{r(x)}$ and this diffeomorphism is required to vary continuously 
with $x$. The same holds for the right multiplication map $R_{y}: x \rightarrow x y$ by Proposition 4 . So the multiplication in $G$ can be regarded as "separately continuous" in the sense that the diffeomorphisms $L_{x}, R_{y}$ 's vary continuously. In contrast, Lie groupoids satisfy the much stronger "joint continuity" condition that the multiplication map $m: G * G \rightarrow G$ is smooth.

It is convenient to have available the pull-back version of (3.2). Note that the pull-back $r^{-1} G$ of $\left.G, r\right)$ is:

$$
\{(x, z) \in G \times G: r(x)=r(z)\} .
$$

The latter set will be denoted by $G *_{r} G$ and is the fibered product $(G, r) *(G, r)$. (Similarly, $G *_{s} G$ is the fibered product $(G, s) *(G, s)$.) The associated morphism $m^{\prime}: G * G \rightarrow G *_{r} G$ is given by: $m^{\prime}(x, y)=(x, x y)$ and we have the diagram:

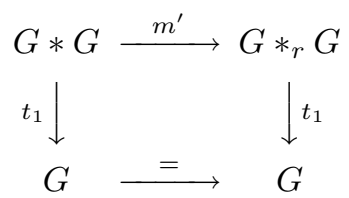

We now discuss some examples of continuous family groupoids. Firstly, every Lie groupoid is a continuous family groupoid. Indeed, in Definition 3, (i) follows since $r, s$ are submersions. Properties (ii) and (iii) follow since both $i$ and $m$ are smooth.

A very simple example of a continuous family groupoid that is not a Lie groupoid is provided by any locally compact Hausdorff space $Y$ that is not a manifold (treated as a groupoid of units). This is the groupoid associated with the (non-equivariant) index theorem for families.

Next, the transformation group groupoid $G$ given by an action of a Lie group $H$ on a locally compact Hausdorff space $Y$ is a continuous family groupoid. In this case, $G=H \times Y$, and the multiplication is given by $\left((h, y),\left(k, k^{-1} y\right)\right) \rightarrow\left(h k, k^{-1} y\right)$ and inversion by $(h, y) \rightarrow\left(h^{-1}, h y\right)$. The unit space $G^{0}$ can be identified with $Y$ and the source and range maps are given by: $s((h, y))=y, r((h, y))=h y$. For $y \in Y$, we have $G_{y}=H, G^{y}=\left\{\left(h, h^{-1} y\right): h \in H\right\}$ which can also be identified with $H$ by sending $\left(h, h^{-1} y\right)$ to $h$. We will not give the proof here that $G$ is a continuous family groupoid since this will be generalized later in Proposition 5. It is easy to see that $G$ is a Lie groupoid if and only if $Y$ is a manifold on which $H$ acts smoothly. This gives many examples of continuous family groupoids which are not Lie groupoids. For example, if $H$ acts trivially on $Y$ and $Y$ is not a manifold, then $G$ is a continuous family groupoid that is not a Lie groupoid.

Another example of a continuous family groupoid is an equivalence relation $G=\left\{\left((y, z),\left(y, z^{\prime}\right)\right): y \in Y, z \in Z\right\}$ on $Y \times Z$, where $Z$ is a smooth manifold. So $G=Y \times(Z \times Z)$ with the product given by $\left(y, z, z^{\prime}\right)\left(y, z^{\prime}, z^{\prime \prime}\right)=\left(y, z, z^{\prime \prime}\right)$ and inversion by $\left(y, z, z^{\prime}\right) \rightarrow\left(y, z^{\prime}, z\right)$. The unit space of $G$ is $Y \times Z$, where $Z$ is identified with the diagonal $\{(z, z): z \in Z\}$ in the obvious way. The source and range maps are given by: $s\left(y, z, z^{\prime}\right)=\left(y, z^{\prime}\right), r\left(y, z, z^{\prime}\right)=(y, z)$. Each of $G^{(y, z)}, G_{(y, z)}$ is just the manifold $Z$. It is easy to check that $G$ is a continuous family groupoid, and is a Lie groupoid if and only if $Y$ is a manifold.

Let $G$ be a continuous family groupoid. From the discussion in $\S 2$ (with $(X, p)=(G, r)$ ), $T G=\cup_{u \in G^{0}} T G^{u}$ is a smooth vector bundle over $(G, r)$. The restriction $A(G)$ of $T G$ to $G^{0}$ is a vector bundle over $G^{0}$, and, as in the case of Lie groupoids, is called the Lie algebroid of $G$. We now briefly discuss the existence of left Haar systems on $G$. The discussion parallels that for Lie groupoids given in $[\mathbf{1 3}, 2.3]$.

For a chart $(U, \phi)$ of (the continuous family) $(G, r)$ and for any $u \in r(U)$, let $\phi^{u}$ be the restriction of $\phi$ to $U \cap G^{u}$ and $W^{u}=\phi^{u}\left(U \cap G^{u}\right) \subset \mathbb{R}^{k}$ (where $k$ is the dimension of the manifolds $G^{u}$ ). Given a measure $\mu$ on $U \cap G^{u}$, the measure $\mu \circ \phi^{u}$ on $W^{u}$ is defined by:

$$
\mu \circ \phi^{u}(E)=\mu\left(\left(\phi^{u}\right)^{-1}(E)\right) .
$$

For any open subset $W$ of $\mathbb{R}^{k}$ let $\lambda^{W}$ be Lebesgue measure restricted to $W$. A left Haar system 
$\left\{\lambda^{u}\right\}$ for $G$ is called a $C^{\infty, 0}$ left Haar system if for any chart $(U, \phi)$ for $(G, r)$, the measure $\lambda^{u} \circ \phi^{u}$ is equivalent to $\lambda^{W^{u}}$, and the map $f$ that sends $(u, t) \rightarrow\left(d\left(\lambda^{u} \circ \phi^{u}\right) / d \lambda^{W^{u}}\right)(u, t)$ belongs to $C^{\infty, 0}(\phi(U))$. The left Haar system is called continuous if $f \in C(\phi(U))$. (Of course every $C^{\infty, 0}$ left Haar system is continuous.) If $G$ is a Lie groupoid, then the left Haar system is called smooth if $f$ is a $C^{\infty}$ function.

Note that two continuous left Haar systems on a continuous family groupoid give isomorphic universal $C^{*}$-algebras and isomorphic reduced $C^{*}$-algebras. The argument for this (which presupposes Renault's representation theory of locally compact groupoids $([\mathbf{9}, \mathbf{1 9}])$ goes as follows. Any quasi-invariant measure $\mu$ on $G^{0}$ determines, for any left Haar system $\left\{\lambda^{u}\right\}$, a measure $\nu=\int \lambda^{u} d \mu(u)$ on $G$, and this in turn determines other measures $\nu^{-1}, \nu^{2}$ on $G$ and $G^{2}$ respectively. Two continuous left Haar systems will give equivalent measures $\nu$ (and similarly for $\nu^{-1}, \nu^{2}$ ) since both $\nu$ 's are equivalent on a chart. The representations for $G$ are then the same for each of these two left Haar systems and any such representation gives equivalent representations for each system on $C_{c}(G)$. It follows that the universal $C^{*}$-algebras $C^{*}(G)$ for the two systems are isomorphic. Similarly, the reduced $C^{*}$-algebras $C_{r e d}^{*}(G)$ are also independent of the choice of system.

In the Lie groupoid case, the equivalence of smooth left Haar systems is captured precisely in Connes's density bundle approach to integration on Lie groupoids ([5, p.101]) which is canonical, and indeed that approach can be readily adapted to apply to $C^{\infty, 0}$ (resp. continuous) left Haar systems for continuous family groupoids. However, for relating the representation theory for continuous family groupoids to the existing representation theory for locally compact groupoids as well as for calculation purposes, the $C^{\infty, 0}$ (resp. continuous) left Haar system approach is convenient and will be used in this paper.

It is known that every Lie groupoid admits a smooth left Haar system. The present writer does not know if every continuous family groupoid admits a $C^{\infty, 0}$ left Haar system. However, as we shall see, every continuous family groupoid admits a continuous left Haar system. The proof of this result is along the same lines as that for the existence of a smooth left Haar system on a Lie groupoid $([\mathbf{1 3}$, p.63]). (See also $[\mathbf{7}, \mathbf{1 7}]$.)

Theorem 1. Let $G$ be a continuous family groupoid. Then there exists a continuous left Haar system on $G$.

Proof. We observe first that the 1-density bundle $\Omega\left(A(G)^{*}\right)$ is trivial, and there exists a strictly positive section $\alpha$ of that bundle. For each $x \in G$, define $L_{x^{-1}}: G^{r(x)} \rightarrow G^{s(x)}$ by: $L_{x^{-1}}(y)=x^{-1} y$. The map $L_{x^{-1}}$ is a diffeomorphism (using Definition 3).

As in the Lie groupoid case, we take the measure $\lambda^{u}$ to be the regular Borel measure associated with the density $z \rightarrow\left(L_{z^{-1}}\right)_{s(z)}^{*}\left(\alpha_{s(z)}\right)$ on $G^{u}$. To check that this is well defined, for each $u_{0} \in G^{0}$ and $z_{0} \in G^{u_{0}}$, we obtain in terms of local charts for $u_{0}, z_{0}$ in $G$ that $\alpha_{u}=g(u) d w_{1} \ldots d w_{k}$ for some continuous positive function $g$, and

$$
\left(L_{z^{-1}}\right)_{s(z)}^{*}\left(g(s(z)) d w_{1} \cdots d w_{k}\right)=g(s(z))\left|J\left(L_{z^{-1}}\right)(z)\right| d z_{1} \cdots d z_{k}
$$

where $J$ stands for the Jacobian. The function $z \rightarrow g(s(z))\left|J\left(L_{z^{-1}}\right)(z)\right|$ is (using Definition 3) a continuous function. It follows that $\left\{\lambda^{u}\right\}$ is a continuous left Haar system on $G$, the proofs of the other items requiring to be checked being the same as in the Lie groupoid case.

\section{Actions of continuous family groupoids}

To motivate the need for continuous family groupoid actions, it is helpful to consider the situation of the Atiyah-Singer equivariant families theorem. (Atiyah and Singer refer this "to the reader" $([\mathbf{2}, \mathrm{p} .135])$.) There, we have a compact fiber bundle $(X, p)$ over $Y$ with compact smooth manifold $Z$ as fiber and structure group $\operatorname{Diff}(Z)$. We are also given a compact Lie group $H$ acting continuously on $Y$ and in a $C^{\infty, 0}$-way on $X$ with $p$ equivariant. We want to 
interpret this in terms of an action of the continuous family groupoid $H * Y$ on $X$, given by:

$$
(h, y) x=h x
$$

where $p(x)=y$. The index of an equivariant family of pseudodifferential operators elliptic along the leaves will then lie in $K_{0}\left(C^{*}(H * Y)\right.$ ). (The Lie groupoid version of this is proved in [14].) So even in the classical Atiyah-Singer index context, we have to leave the category of groups and move to the category of continuous family groupoids.

With this motivation, we now turn to the problem of determining what a left action of a continuous family groupoid should be. It turns out - slightly surprisingly - that all that we require for present purposes is a continuous action of a continuous family groupoid $G$ on a fiber space $Y$ over $G^{0}$. The reason is that the unit space of the locally compact groupoid $G * Y$ is identified with $Y$, while its fibers $(G * Y)_{y},(G * Y)^{y}$ are effectively the continuous family groupoid fibers $G_{p(y)}, G^{p(y)}$, so that the $C^{\infty, 0}$ - structure of $G * Y$ is effectively that of $G$ with the $Y$ only playing the role of a continuous parameter space. We now discuss this in more detail.

Let $G$ be a locally compact groupoid (not necessarily a continuous family groupoid) with $r, s$ open maps, and let $(Y, p)$ be a fiber space over $G^{0}$. Form the fibered product $G * Y=$ $(G, s) *(Y, p)$ of the fiber spaces $(G, s),(Y, p)$ over $G^{0}$. The action of $G$ on $Y$ is then given by a continuous map $n: G * Y \rightarrow Y$. The action has to satisfy the natural algebraic axioms: so we require $p(g y)=r(g), g_{1}\left(g_{2} y\right)=\left(g_{1} g_{2}\right) y$ and $g^{-1}(g y)=y$ whenever these make sense. The space $Y$ with such an action of $G$ is called a $G$-space (cf.[10]).

Let $Y$ be a $G$-space. It is well known and easy to check from the groupoid axioms of $\S 2$ that $G * Y$ is a locally compact groupoid with operations given by: $(h, z)\left(g, g^{-1} z\right)=\left(h g, g^{-1} z\right)$ and $(g, y)^{-1}=\left(g^{-1}, g y\right)$. Let $\mu$ be the multiplication map and $i$ be the inversion map on $G * Y$. So

$$
\mu\left(h, z, g, g^{-1} z\right)=\left(h g, g^{-1} z\right) .
$$

Further, $s(g, y)=(s(g), y), r(g, y)=(r(g), g y)$. We can identify $(s(g), y)$ with $y$ (since $s(g)=$ $p(y))$, and so $(G * Y)^{0}=Y$. (In fact, this is just identifying $G^{0} * Y$ with $Y$.) Note that $r=n$ and $s=t_{2}$.

Now, in addition, assume that $G$ is a continuous family groupoid. We will show that $G * Y$ is also, in a natural way, a continuous family groupoid. We first discuss the continuous family structures for $(G * Y, s)$ and $(G * Y, r)$. We have a commuting diagram:

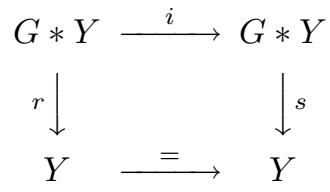

where $i$ is the inversion map on $G * Y$. We can identify $(G * Y, s)$ canonically with $\left(G * Y, t_{2}\right)$. As observed in $\S 2,\left(G * Y, t_{2}\right)$ is a continuous family (since $(G, s)$ is a continuous family). Next, the map $r$ is a continuous surjection which is open, since $s$ is and $i$ is a homeomorphism. We give $(G * Y, r)$ the (unique) continuous family structure that makes $i$ an isomorphism of continuous families. In particular, an atlas for $(G * Y, r)$ is determined by charts of the form $i^{-1}(U * V)$, and the $C^{\infty}$-structure on each $(G * Y)^{z}=\left\{\left(g, g^{-1} z\right): g \in G^{p(z)}\right\}$ is just that obtained by identifying $(G * Y)^{z}$ with $G_{p(z)}$ through the map $\left(g, g^{-1} z\right) \rightarrow g^{-1}$ (and hence with $G^{p(z)}$ through the map $\left(g, g^{-1} z\right) \rightarrow g$, using (ii) of Definition 3.)

Proposition 5. The continuous family $G * Y=(G, s) *(Y, p)$ is a continuous family groupoid.

Proof. We check that the requirements for a continuous family groupoid in Definition 3 hold for $G * Y$. (i) and (ii) of that definition follow from the discussion preceding the statement of the proposition. It remains to show that the multiplication map $\mu$ on $G * Y$ is a morphism. 
To this end, consider the following commutative diagram:

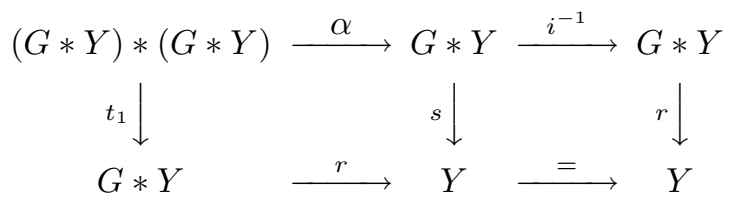

Here, $\alpha\left(h, z, g, g^{-1} z\right)=\left(g^{-1} h^{-1}, h z\right)$. Then $\mu=i^{-1} \circ \alpha$, and since $i^{-1}$ is a morphism $((4.2))$, it remains to show that $\alpha$ is a morphism. It is sufficient for this to show that $\gamma: G * G \rightarrow G$, where $\gamma(h, g)=g^{-1} h^{-1}$, is a morphism:

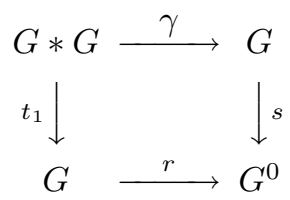

For since the map $(h, z) \rightarrow h z$ is continuous, it follows that $(h, z) \rightarrow \alpha_{(h, z)}$ varies in a $C^{\infty, 0} 0_{-}$ way if the map $h \rightarrow \gamma_{h}$ does. The map $\gamma$ is a morphism since it is the composition of two morphisms:

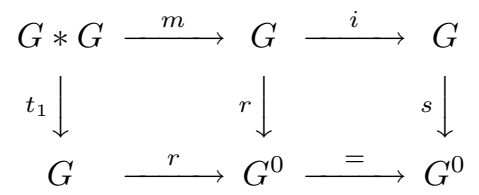

Motivated by the families situation (discussed in the first paragraph of this section) we now have to extend the $G$-space notion to that of a fiber space $(X, q)$ over $Y$ which is also a $G$-space with an action compatible with that on $Y$. compatible action. Precisely, $(X, p \circ q)$ is a fiber space over $G^{0}$ and we require that this fiber space and $(Y, p)$ be $G$-spaces such that for all $(g, x) \in G * X$, we have $q(g x)=g q(x)$. (Note that $(g, q(x)) \in G * Y$ since $p(q(x))=s(g)$.) This can be formulated in terms of the commuting diagram of continuous maps:

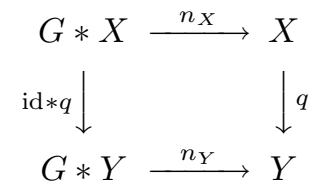

where $n_{X}, n_{Y}$ are the action maps of $G$ on $X$ and $Y$ respectively. We say that $X$ is a $G$-space over $Y$. We now show that $X$ is itself a $G * Y$-space in a natural way.

Theorem 2. Let $G$ be a continuous family groupoid and $Y$ be a $G$-space. Then the class of $G$-spaces $X$ over $Y$ is canonically identified with the class of $G * Y$-spaces.

Proof. Let $(X, q)$ be a $G$-space over $Y$. Recalling that the source map of the groupoid $G * Y$ is the map $(g, y) \rightarrow y$, it follows that $(G * Y) * X=(G * Y, s) *(X, q)$ is a fiber space over $Y$. Define a map

$$
n_{Y, X}:(G * Y) * X \rightarrow X
$$

by: $n_{Y, X}(g, y, x)=g x$. Calculations very similar to those of the next paragraph show that $n_{Y, X}$ is an action of $G * Y$ on $X$. So $X$ is a $G * Y$-space.

Conversely, suppose that $(X, q)$ is a $G * Y$-space. Then $q: X \rightarrow(G * Y)^{0}=Y$ is continuous and onto, and so $X$ is a fiber space over $Y$. Let $n:(G * Y) * X \rightarrow X$ be the action map. Then $(X, p \circ q)$ is a fiber space over $Y$, so that $G * X$ is defined. For $(g, x) \in G * X$, define the action of $G$ on $X$ by:

$$
g x=n((g, q(x)), x)=(g, q(x)) x .
$$


Clearly the map $(g, x) \rightarrow g x$ is continuous. We now check the algebraic action axioms for the map $(g, x) \rightarrow g x$. For the associative law, with $z=q(x)$, we have $(h g) x=[(h, g z)(g, z)] x=$ $(h, g z)[(g, z) x]=(h, g z)(g x)=h(g x)$. Next $g^{-1}(g x)=s(g) x=s((g, z)) x=x$. Lastly, $q(g x)=$ $q((g, z) x)=r((g, z))=g z$. It follows that $X$ is a $G$-space over $Y$. It is left to the reader to show that if we apply the $n_{Y, X}$ construction to $X$ with this $G$-space structure, then we get back to the $G * Y$-space with which we started.

In the situation of the equivariant families index theorem, one has to consider a $G$-space $X$ over $Y$ which is a continuous family and on which $G$ acts in a $C^{\infty, 0}-$ way. We now briefly indicate how this is defined. By Theorem 2, we can, by replacing $G$ by $G * Y$, suppose that $X=$ $Y$. Then the action of $G$ on $Y$ is said to be $C^{\infty, 0}$ if the multiplication map $n:\left(G * Y, t_{1}\right) \rightarrow Y$ is a morphism with respect to $r$ :

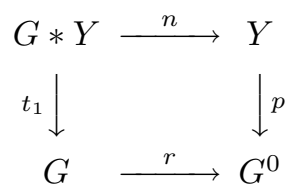

We say that $Y$ is a $C^{\infty, 0} G$-space. The continuous family groupoid $G$ is itself a $C^{\infty, 0} G$-space. This follows from (iii) of Definition 3.

As in (3.5), the morphism property of (4.8) can be reformulated in terms of a morphism $n^{\prime}$ :

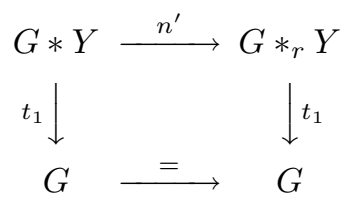

where $G *_{r} Y=(G, r) *(Y, p)$ and the map $n^{\prime}$ is given by: $n^{\prime}(g, y)=(g, g y)$.

In conclusion, Theorem 2 says that a $G$-space $X$ over $Y$ is the same as a $(G * Y)$-space, the fibering of $X$ over $Y$ being "absorbed" as it were into the fibering of the continuous family groupoid $G * Y$. (The same applies if we work in the category of $C^{\infty, 0} G$-spaces.) Note that this cannot be formulated if we stay within the group category, since in forming $G * Y$, we leave the group category. So we do not need to work in the situation where a $G$-space $X$ is fibered over another $G$-space $Y$. For the "higher order" fibered space $X$ is itself just an "ordinary" groupoid space for the groupoid $G * Y$, and we do not leave the category of continuous family groupoids by forming $G * Y$. Thus we only ever need consider the action of a continuous family groupoid on a $G$-space, changing the groupoid if necessary.

\section{References}

[1] M. F. Atiyah and I. Singer, The index of elliptic operators, I, Ann. of Math. 87(1968), 484-530.

[2] M. F. Atiyah and I. Singer, The index of elliptic operators, IV, Ann. of Math. 93(1971), $119-38$.

[3] C. Camacho and A. L. Neto, Geometric Theory of Foliations, Birkhäuser, Boston, 1985.

[4] A. Connes, Sur la théorie non commutative de l'intégration, Lecture Notes in Mathematics, 725(1979), 19-143.

[5] A. Connes, Noncommutative Geometry, Academic Press, Inc., New York, 1994.

[6] S. Kobayashi and K. Nomizu, Foundations of Differential Geometry, Vol. 1, Interscience Tracts, No. 15, John Wiley and Sons, New York, 1963.

[7] N. P. Landsman, Mathematical Topics between Classical and Quantum Mechanics, Springer-Verlag, New York, 1998. 
[8] K. C. H. Mackenzie, Lie Groupoids and Lie algebroids in Differential Geometry, London Mathematical Society Lecture Note Series, vol. 124, Cambridge University Press, Cambridge, 1987.

[9] P. S. Muhly, Coordinates in Operator Algebra, to appear, CBMS Regional Conference Series in Mathematics, American Mathematical Society, Providence, 180pp..

[10] P. S. Muhly, J. N. Renault and D. P. Williams, Equivalence and isomorphism for groupoid $C^{*}$-algebras, J. Operator Theory 17(1987), 3-22.

[11] P. S. Muhly and D. P. Williams, Groupoid cohomology and the Dixmier-Douady class. Proc. London Math. Soc. 71(1995), 109-134.

[12] V. Nistor, A. Weinstein and P. Xu, Pseudodifferential operators on differential groupoids, Pacific J. Math. 189(1999), 117-152.

[13] A. L. T. Paterson, Groupoids, inverse semigroups and their operator algebras, Progress in Mathematics, Vol. 170, Birkhäuser, Boston, 1999.

[14] A. L. T. Paterson, The analytic index for proper, Lie groupoid actions, preprint, 1999.

[15] J. Pradines, Théorie de Lie pour les groupoïdes différentiables. Relations entre propriétés locales et globales. C. R. Acad. Sci. Paris Sér. A-B 263(1966), A907-A910.

[16] J. Pradines, Théorie de Lie pour les groupoïdes différentiables. Calcul différential dans la catégorie des groupoïdes infinitésimaux, C. R. Acad. Sci. Paris Sér. A-B 264(1967), A 245-A 248.

[17] B. Ramazan, Deformation Quantization of Lie-Poisson Manifolds, Ph. D. Thesis, Université d'Orléans, 1998.

[18] J. N. Renault, A groupoid approach to $C^{*}$-algebras, Lecture Notes in Mathematics, Vol. 793, Springer-Verlag, New York, 1980.

[19] J. N. Renault, Répresentation de produits croisés d'algèbres de groupoïdes, J. Operator Theory, 18(1987), 67-97.

Alan L. T. Paterson mmap@olemiss.edu

Department of Mathematics

University of Mississippi

University, MS 38677 